\title{
Spaces of real polynomials with common roots
}

\author{
YASUHIKO KAMIYAMA
}

Let $R X_{k, n}^{l}$ be the space consisting of all $(n+1)$-tuples $\left(p_{0}(z), \ldots, p_{n}(z)\right)$ of monic polynomials over $\mathbb{R}$ of degree $k$ and such that there are at most $l$ roots common to all $p_{i}(z)$. In this paper, we prove a stable splitting of $R X_{k, n}^{l}$.

55P15; 55P35, 58D15

\section{Introduction}

Let $\operatorname{Rat}_{k}\left(\mathbb{C} P^{n}\right)$ denote the space of based holomorphic maps of degree $k$ from the Riemannian sphere $S^{2}=\mathbb{C} \cup \infty$ to the complex projective space $\mathbb{C} P^{n}$. The basepoint condition we assume is that $f(\infty)=[1, \ldots, 1]$. Such holomorphic maps are given by rational functions:

$\operatorname{Rat}_{k}\left(\mathbb{C} P^{n}\right)=\left\{\left(p_{0}(z), \ldots, p_{n}(z)\right)\right.$ : each $p_{i}(z)$ is a monic polynomial over $\mathbb{C}$ of degree $k$ and such that there are no roots common to all $\left.p_{i}(z)\right\}$.

There is an inclusion $\operatorname{Rat}_{k}\left(\mathbb{C} P^{n}\right) \hookrightarrow \Omega_{k}^{2} \mathbb{C} P^{n} \simeq \Omega^{2} S^{2 n+1}$. Segal [6] proved that the inclusion is a homotopy equivalence up to dimension $k(2 n-1)$. Later, the stable homotopy type of $\operatorname{Rat}_{k}\left(\mathbb{C} P^{n}\right)$ was described by Cohen et al [2;3] as follows. Let $\Omega^{2} S^{2 n+1} \underset{s}{\simeq} \bigvee_{1 \leq q} D_{q}\left(S^{2 n-1}\right)$ be Snaith's stable splitting of $\Omega^{2} S^{2 n+1}$. Then

$$
\operatorname{Rat}_{k}\left(\mathbb{C} P^{n}\right) \underset{s}{\simeq} \bigvee_{q=1}^{k} D_{q}\left(S^{2 n-1}\right) .
$$

In Kamiyama [4], (1-1) was generalized as follows. We set

$X_{k, n}^{l}=\left\{\left(p_{0}(z), \ldots, p_{n}(z)\right):\right.$ each $p_{i}(z)$ is a monic polynomial over $\mathbb{C}$

of degree $k$ and such that there are at most $l$ roots common to all $\left.p_{i}(z)\right\}$.

In particular, $X_{k, n}^{0}=\operatorname{Rat}_{k}\left(\mathbb{C} P^{n}\right)$. Let

$$
J^{l}\left(S^{2 n}\right) \simeq S^{2 n} \cup e^{4 n} \cup e^{6 n} \cup \ldots \cup e^{2 l n} \subset \Omega S^{2 n+1}
$$


be the $l$ th stage of the James filtration of $\Omega S^{2 n+1}$, and let $W^{l}\left(S^{2 n}\right)$ be the homotopy theoretic fiber of the inclusion $J^{l}\left(S^{2 n}\right) \hookrightarrow \Omega S^{2 n+1}$. We generalize Snaith's stable splitting of $\Omega^{2} S^{2 n+1}$ as follows:

$$
W^{l}\left(S^{2 n}\right) \underset{s}{\simeq} \bigvee_{1 \leq q} D_{q} \xi^{l}\left(S^{2 n}\right)
$$

Then we have a stable splitting

$$
X_{k, n}^{l} \underset{s}{\sim} \bigvee_{q=1}^{k} D_{q} \xi^{l}\left(S^{2 n}\right) .
$$

The purpose of this paper is to study the real part $R X_{k, n}^{l}$ of $X_{k, n}^{l}$ and prove a stable splitting of this. More precisely, let $R X_{k, n}^{l}$ be the subspace of $X_{k, n}^{l}$ consisting of elements $\left(p_{0}(z), \ldots, p_{n}(z)\right)$ such that each $p_{i}(z)$ has real coefficients. Our main results will be stated in Section 2. Here we give a theorem which generalizes (1-1). Since the homotopy type of $R X_{k, 1}^{0}$ is known (see Example 2.1 (iii)), we assume $n \geq 2$. In this case, there is an inclusion

$$
R X_{k, n}^{0} \hookrightarrow \Omega S^{n} \times \Omega^{2} S^{2 n+1}
$$

(See Lemma 3.1.)

Theorem 1.1 For $n \geq 2$, we define the weight of stable summands in $\Omega S^{n}$ as usual, but those in $\Omega^{2} S^{2 n+1}$ we define as being twice the usual one. Then $R X_{k, n}^{0}$ is stably homotopy equivalent to the collection of stable summands in $\Omega S^{n} \times \Omega^{2} S^{2 n+1}$ of weight $\leq k$. Hence,

$$
R X_{k, n}^{0} \simeq \bigvee_{p+2 q \leq k} \Sigma^{p(n-1)} D_{q}\left(S^{2 n-1}\right) \vee \bigvee_{p=1}^{k} S^{p(n-1)}
$$

This paper is organized as follows. In Section 2 we state the main results. We give a stable splitting of $R X_{k, n}^{l}$ in Theorem A and Theorem B. In order to prove these theorems, we also consider a space $Y_{k, n}^{l}$, which is an open set of $R X_{k, n}^{l}$. We give a stable splitting of $Y_{k, n}^{l}$ in Proposition C. In Section 3 we prove Proposition C. In Section 4 we prove Theorem A and Theorem B. 


\section{Main results}

We set

$$
Y_{k, n}^{l}=\left\{\left(p_{0}(z), \ldots, p_{n}(z)\right) \in R X_{k, n}^{l}: \text { there are no real roots common to all } p_{i}(z)\right\} \text {. }
$$

The spaces $Y_{k, n}^{l}$ and $R X_{k, n}^{l}$ are in the following relation:

$$
\begin{array}{ccccccccccccc}
Y_{k, n}^{k} & \supset & Y_{k, n}^{k-1} & \supset & \cdots & \supset & Y_{k, n}^{l} & \supset & \cdots & \supset & Y_{k, n}^{1}= & Y_{k, n}^{0} \\
\cap & & \cap & & & & \cap & & & \cap & \| \\
R X_{k, n}^{k} & \supset & R X_{k, n}^{k-1} & \supset & \cdots & \supset & R X_{k, n}^{l} & \supset & \cdots & \supset & R X_{k, n}^{1} & \supset & R X_{k, n}^{0}
\end{array}
$$

where each subset is an open set. Moreover, $Y_{k, n}^{2 i+1}=Y_{k, n}^{2 i}$. In fact, if $\alpha \in H_{+}$(where $H_{+}$is the open upper half-plane) is a root of a real polynomial, then so is $\bar{\alpha} \in H_{-}$.

We have the following examples.

\section{Example 2.1}

(i) It is proved by Mostovoy [5] that $Y_{k, 1}^{k}$ consists of $k+1$ contractible connected components.

(ii) The following result is proved by Vassiliev [7]. For $n \geq 3$, there is a homotopy equivalence $Y_{k, n}^{k} \simeq J^{k}\left(S^{n-1}\right)$, where $J^{k}\left(S^{n-1}\right)$ is as above the $k$ th stage of the James filtration of $\Omega S^{n}$. For $n=2$, these spaces are stably homotopy equivalent.

(iii) It is proved by Segal [6] that

$$
R X_{k, 1}^{0} \simeq \coprod_{q=0}^{k} \operatorname{Rat}_{\min (q, k-q)}\left(\mathbb{C} P^{1}\right) .
$$

(iv) $R X_{k, n}^{k-1} \cong \mathbb{R}^{k} \times\left(\mathbb{R}^{k n}\right)^{*}$ and $R X_{k, n}^{k} \cong \mathbb{R}^{k(n+1)}$.

In fact, $\left(p_{0}(z), \ldots, p_{n}(z)\right) \in R X_{k, n}^{k}$ is an element of $R X_{k, n}^{k-1}$ if and only if $p_{i}(z) \neq$ $p_{j}(z)$ for some $i, j$. Hence, the first homeomorphism holds.

Now we state our main results.

Theorem A For $n \geq 1$ and $i \geq 0$, there is a homotopy equivalence

$$
R X_{k, n}^{2 i+1} \simeq X_{\left[\frac{k}{2}\right], n}^{i},
$$

where $\left[\frac{k}{2}\right]$ denotes as usual the largest integer $\leq \frac{k}{2}$. 
Theorem B For $n \geq 1$ and $i \geq 0$, there is a stable homotopy equivalence

$$
R X_{k, n}^{2 i} \underset{s}{\simeq} X_{\left[\frac{k}{2}\right], n}^{i} \vee \Sigma^{2 i n}\left(\bigvee_{\substack{p+2 q \leq k-2 i \\ 1 \leq p}} \Sigma^{p(n-1)} D_{q}\left(S^{2 n-1}\right) \vee \bigvee_{p=1}^{k-2 i} S^{p(n-1)}\right)
$$

We study $R X_{k, n}^{l}$ by induction with making $l$ larger. Hence, the induction starts from $R X_{k, n}^{0}$. Recall that $R X_{k, n}^{0}=Y_{k, n}^{0}$. We study $Y_{k, n}^{l}$ by induction with making $l$ smaller, where the initial condition is given in Example 2.1 (ii). In fact, we have the following proposition.

\section{Proposition C}

(i) For $n \geq 2$, we define the weight of stable summands in $\Omega S^{n}$ as usual, but those in $W^{i}\left(S^{2 n}\right)$ we define as being twice the usual one. Then $Y_{k, n}^{2 i}$ is stably homotopy equivalent to the collection of stable summands in $\Omega S^{n} \times W^{i}\left(S^{2 n}\right)$ of weight $\leq k$. Hence,

$$
Y_{k, n}^{2 i} \underset{s}{\simeq} \bigvee_{p+2 q \leq k} \Sigma^{p(n-1)} D_{q} \xi^{i}\left(S^{2 n}\right) \vee \bigvee_{p=1}^{k} S^{p(n-1)} .
$$

(ii) When $n=1$, there is a homotopy equivalence

$$
Y_{k, 1}^{2 i} \simeq \coprod_{q=0}^{k} X_{\min (q, k-q), 1}^{i} .
$$

Note that Proposition C (ii) is a generalization of Example 2.1 (i) and (iii).

\section{Proof of Proposition C}

We study the space of continuous maps which contains $Y_{k, n}^{k}$ or $R X_{k, n}^{0}$. For simplicity, we assume $n \geq 2$. (The case for $n=1$ can be obtained by slight modifications.) Each $f \in Y_{k, n}^{k}$ defines a map $f: S^{1} \rightarrow \mathbb{R} P^{n}$, where $S^{1}=\mathbb{R} \cup \infty$. Hence, there is a natural map

$$
Y_{k, n}^{k} \rightarrow \Omega_{k \bmod 2} \mathbb{R} P^{n} \simeq \Omega S^{n} .
$$

Example 2.1 (ii) implies that $Y_{k, n}^{k}$ is the $k(n-1)$-skeleton of $\Omega S^{n}$. 
On the other hand, let $\operatorname{Map}_{k}^{T}\left(\mathbb{C} P^{1}, \mathbb{C} P^{n}\right)$ be the space of continuous basepointpreserving conjugation-equivariant maps of degree $k$ from $\mathbb{C} P^{1}$ to $\mathbb{C} P^{n}$. Then there is an inclusion

$$
R X_{k, n}^{0} \hookrightarrow \operatorname{Map}_{k}^{T}\left(\mathbb{C} P^{1}, \mathbb{C} P^{n}\right)
$$

Lemma 3.1 For $n \geq 2, \operatorname{Map}_{k}^{T}\left(\mathbb{C} P^{1}, \mathbb{C} P^{n}\right) \simeq \Omega S^{n} \times \Omega^{2} S^{2 n+1}$.

Proof It is easy to see that

$$
\operatorname{Map}_{k}^{T}\left(\mathbb{C} P^{1}, \mathbb{C} P^{n}\right) \simeq \operatorname{Map}_{0}^{T}\left(\mathbb{C} P^{1}, \mathbb{C} P^{n}\right) .
$$

Since $\operatorname{Map}_{0}^{T}\left(\mathbb{C} P^{1}, \mathbb{C} P^{n}\right)$ can be thought as the space of maps

$$
\left(D^{2}, S^{1}, *\right) \rightarrow\left(\mathbb{C} P^{n}, \mathbb{R} P^{n}, *\right)
$$

of degree 0 , there is a fibration

$$
\Omega^{2} S^{2 n+1} \rightarrow \operatorname{Map}_{0}^{T}\left(\mathbb{C} P^{1}, \mathbb{C} P^{n}\right) \rightarrow \Omega S^{n} .
$$

This is a pullback of the path fibration $\Omega^{2} S^{2 n+1} \rightarrow P \Omega S^{2 n+1} \rightarrow \Omega S^{2 n+1}$ by the map $\Omega f: \Omega S^{n} \rightarrow \Omega S^{2 n+1}$, where $f: S^{n} \rightarrow S^{2 n+1}$ is a lift of the inclusion $\mathbb{R} P^{n} \hookrightarrow \mathbb{C} P^{n}$. Since $f$ is null homotopic, the fibration is trivial. This completes the proof of Lemma 3.1 .

Hereafter, every homology is with $\mathbb{Z} / p$-coefficients, where $p$ is a prime. Recall that for $n \geq 2$, we have $H_{*}\left(\Omega S^{n}\right) \cong \mathbb{Z} / p\left[x_{n-1}\right]$. We define the weight of $x_{n-1}$ by $w\left(x_{n-1}\right)=1$. On the other hand, we define the weight of an element of $H_{*}\left(X_{k, n}^{i}\right)$ as being twice the usual one. For example, let $y_{2(l+1) n-1}$ be the generator of $\widetilde{H}_{*}\left(X_{k, n}^{l}\right)$ of least degree. The usual weight of $y_{2(l+1) n-1}$ is $l+1$, but we reset $w\left(y_{2}(l+1) n-1\right)=$ $2(l+1)$.

Proposition 3.2 For $n \geq 2, H_{*}\left(Y_{k, n}^{2 i}\right)$ is isomorphic to the subspace of $H_{*}\left(\Omega S^{n} \times X_{k, n}^{i}\right)$ spanned by monomials of weight $\leq k$.

We prove the proposition from the following lemma.

Lemma 3.3 We have the following long exact sequence:

$$
\cdots \rightarrow H_{*}\left(Y_{k, n}^{2 i-2}\right) \rightarrow H_{*}\left(Y_{k, n}^{2 i}\right) \stackrel{\phi}{\rightarrow} H_{*-2 i n}\left(R X_{k-2 i, n}^{0}\right) \rightarrow H_{*-1}\left(Y_{k, n}^{2 i-2}\right) \rightarrow \cdots
$$


Proof In [4, Propositions 4.5 and 5.4], we constructed a similar long exact sequence from the fact that

$$
X_{k, n}^{l}-X_{k, n}^{l-1}=\mathbb{C}^{l} \times \operatorname{Rat}_{k-l}\left(\mathbb{C} P^{n}\right),
$$

where $\mathbb{C}^{l} \times$ Rat $_{k-l}\left(\mathbb{C} P^{n}\right)$ corresponds to the subspace of $X_{k, n}^{l}$ consisting of elements $\left(p_{0}(z), \ldots, p_{n}(z)\right)$ such that there are exactly $l$ roots common to all $p_{i}(z)$. The proposition is proved similarly using the fact that

$$
Y_{k, n}^{2 i}-Y_{k, n}^{2 i-2} \cong \mathrm{SP}^{i}\left(H_{+}\right) \times R X_{k-2 i, n}^{0},
$$

where $\mathrm{SP}^{i}\left(H_{+}\right)$denotes the $i$ th symmetric product of $H_{+}$.

Proof of Proposition 3.2 In order to prove Proposition 3.2 by induction, we introduce the following total order $\leq$ to $Y_{k, n}^{2 i}$ for $k \geq 1$ and $i \geq 0: Y_{k, n}^{2 i}<Y_{k^{\prime}, n}^{2 i^{\prime}}$ if and only if

(i) $k<k^{\prime}$, or

(ii) $k=k^{\prime}$ and $i>i^{\prime}$.

By Example 2.1 (ii), Proposition 3.2 holds for $Y_{k, n}^{k}$. Assuming that Proposition 3.2 holds for $Y_{k, n}^{2 i}$ and $R X_{k-2 i, n}^{0}$, we prove for $Y_{k, n}^{2 i-2}$. We have the following long exact sequence:

$$
\begin{aligned}
(3-1) \quad \cdots \longrightarrow H_{*}\left(X_{\left[\frac{k}{2}\right], n}^{i-1}\right) & \longrightarrow H_{*}\left(X_{\left[\frac{k}{2}\right], n}^{i}\right) \\
\stackrel{\psi}{\longrightarrow} & H_{*-2 i n}\left(\operatorname{Rat}_{\left[\frac{k}{2}\right]-i}\left(\mathbb{C} P^{n}\right)\right) \stackrel{\theta}{\longrightarrow} H_{*-1}\left(X_{\left[\frac{k}{2}\right], n}^{i-1}\right) \longrightarrow \cdots .
\end{aligned}
$$

For $n \geq 2$, we consider the homomorphism

$$
1 \otimes \psi: H_{*}\left(\Omega S^{n}\right) \otimes H_{*}\left(X_{\left[\frac{k}{2}\right], n}^{i}\right) \rightarrow H_{*}\left(\Omega S^{n}\right) \otimes H_{*-2 i n}\left(\operatorname{Rat}_{\left[\frac{k}{2}\right]-i}\left(\mathbb{C} P^{n}\right)\right) .
$$

Restricting the domain to $H_{*}\left(Y_{k, n}^{2 i}\right)$, we obtain the homomorphism $\phi$ in Lemma 3.3. Now it is easy to prove Proposition 3.2.

Proof of Proposition C (i) We construct a stable map from the right-hand side of Proposition C (i) to $Y_{k, n}^{2 i}$. Since our constructions are similar, we construct a stable map $g_{p, q, i, n}: \Sigma^{p(n-1)} D_{q} \xi^{i}\left(S^{2 n}\right) \rightarrow Y_{k, n}^{2 i}$. First, using the fact that $R X_{1, n}^{0} \simeq S^{n-1}$ (see Example 2.1 (iv)), there is a stable map $f_{p, n}: S^{p(n-1)} \rightarrow R X_{p, n}^{0}$. Second, there is a stable section $e_{q, i, n}: D_{q} \xi^{i}\left(S^{2 n}\right) \rightarrow X_{q, n}^{i}$. Third, there is an inclusion

$$
\eta_{q, i, n}: X_{q, n}^{i} \hookrightarrow Y_{2 q, n}^{2 i} .
$$


To construct this, we fix a homeomorphism $h: \mathbb{C} \cong H_{+}$. For $\left(p_{0}(z), \ldots, p_{n}(z)\right)$ $\in X_{q, n}^{i}$, we write $p_{j}(z)=\prod_{s=1}^{q}\left(z-\alpha_{s, j}\right)$. Then we set

$$
\begin{aligned}
\eta_{q, i, n}\left(p_{0}(z), \ldots, p_{n}(z)\right) & \\
& =\left(\prod_{s=1}^{q}\left(z-h\left(\alpha_{s, 0}\right)\right)\left(z-\overline{h\left(\alpha_{s, 0}\right)}\right), \ldots, \prod_{s=1}^{q}\left(z-h\left(\alpha_{s, n}\right)\right)\left(z-\overline{h\left(\alpha_{s, n}\right)}\right)\right) .
\end{aligned}
$$

Now consider the following composite of maps

$$
S^{p(n-1)} \times D_{q} \xi^{i}\left(S^{2 n}\right) \stackrel{f_{p, n} \times\left(\eta_{q, i, n} \circ e_{q, i, n}\right)}{\longrightarrow} R X_{p, n}^{0} \times Y_{2 q, n}^{2 i} \stackrel{\mu}{\rightarrow} Y_{p+2 q, n}^{2 i} \hookrightarrow Y_{k, n}^{2 i},
$$

where $\mu$ is a loop sum which is constructed in the same way as in the loop sum $\operatorname{Rat}_{k}\left(\mathbb{C} P^{n}\right) \times \operatorname{Rat}_{l}\left(\mathbb{C} P^{n}\right) \rightarrow \operatorname{Rat}_{k+l}\left(\mathbb{C} P^{n}\right)$ in Boyer-Mann [1]. We can construct $g_{p, q, i, n}$ from (3-3).

Note that the stable map for Proposition C (i) is compatible with the homology splitting by weights. Using Proposition 3.2, it is easy to show that this map induces an isomorphism in homology, hence is a stable homotopy equivalence. This completes the proof of Proposition C (i).

Proof of Proposition C (ii) By a similar argument to the proof of Proposition 3.2, we can calculate $H_{*}\left(Y_{k, 1}^{2 i}\right)$. Then we can construct an unstable map from the right-hand side of Proposition C (ii) to $Y_{k, 1}^{2 i}$ in the same way as in Proposition C (i).

\section{Proof of Theorem A and Theorem B}

Proposition 4.1 The homologies of the both sides of Theorem A or Theorem B are isomorphic.

Proof We prove the proposition about $R X_{k, n}^{l}$ by induction with making $l$ larger. As in Lemma 3.3, there is a long exact sequence

$$
\begin{aligned}
\cdots \longrightarrow H_{*}\left(R X_{k, n}^{l}\right) \longrightarrow & H_{*}\left(R X_{k, n}^{l+1}\right) \\
& \longrightarrow H_{*-(l+1) n}\left(R X_{k-(l+1), n}^{0}\right) \stackrel{\Theta}{\longrightarrow} H_{*-1}\left(R X_{k, n}^{l}\right) \longrightarrow \cdots .
\end{aligned}
$$

This sequence is constructed from the following decomposition as sets

$$
R X_{k, n}^{l+1}-R X_{k, n}^{l}=\coprod_{a+2 b=l+1} \operatorname{SP}^{a}(\mathbb{R}) \times \operatorname{SP}^{b}\left(H_{+}\right) \times R X_{k-(l+1), n}^{0}
$$


and the fact that $H_{c}^{*}\left(\mathrm{SP}^{a}(\mathbb{R})\right)=0$ for $a \geq 2$, where $H_{c}^{*}$ is the cohomology with compact supports.

Assuming that the proposition holds for $l \leq 2 i+1$, we determine $H_{*}\left(R X_{k, n}^{2 i+2}\right)$. The homomorphism $\Theta$ is given as follows. Note that Theorem B is equivalent to

$$
R X_{k, n}^{2 i} \underset{s}{\simeq} X_{\left[\frac{k}{2}\right], n}^{i} \vee \Sigma^{(2 i+1) n-1}\left(R X_{k-2 i-1, n}^{0} \vee S^{0}\right) .
$$

From inductive hypothesis, we have

$$
\begin{aligned}
& H_{*-(2 i+2) n}\left(R X_{k-2 i-2, n}^{0}\right) \cong \\
& H_{*-(2 i+2) n}\left(\operatorname{Rat}_{\left[\frac{k}{2}\right]-(i+1)}\left(\mathbb{C} P^{n}\right)\right) \oplus \widetilde{H}_{*-(2 i+2) n}\left(\Sigma^{n-1} R X_{k-2 i-3, n}^{0} \vee S^{n-1}\right)
\end{aligned}
$$

and

$$
H_{*-1}\left(R X_{k, n}^{2 i+1}\right) \cong H_{*-1}\left(X_{\left[\frac{k}{2}\right], n}^{i}\right) .
$$

Recall the homomorphism $\theta$ in (3-1) with $i$ replaced by $i+1$. Then $\Theta$ : (4-2) $\rightarrow$ (4-3) is given by mapping the first summand by $\theta$ and the second summand by 0 . Hence, $H_{*}\left(R X_{k, n}^{2 i+2}\right)$ is isomorphic to the homology of the right-hand side of (4-1) with $i$ replaced by $i+1$.

By a similar argument, we can determine $H_{*}\left(R X_{k, n}^{2 i+1}\right)$ inductively by assuming the truth of the proposition for $l \leq 2 i$. This completes the proof of Proposition 4.1.

Finally, we construct an unstable map (resp. a stable map) from the right-hand side of Theorem A (resp. (4-1)) to $R X_{k, n}^{2 i+1}$ (resp. $R X_{k, n}^{2 i}$ ). First, the unstable map from the right-hand side of Theorem $A$ or the first stable summand in (4-1) is essentially the inclusion

$$
X_{q, n}^{i} \stackrel{\eta_{q, i, n}}{\longrightarrow} Y_{2 q, n}^{2 i} \subset R X_{2 q, n}^{2 i},
$$

where $\eta_{q, i, n}$ is defined in (3-2). Next, the stable map from the second stable summand in (4-1) is constructed in the same way as in $g_{p, q, i, n}$ (see (3-3)) using the fact that $R X_{2 i+1, n}^{2 i} \simeq S^{(2 i+1) n-1}$ (see Example 2.1 (iv)). This completes the proofs of Theorem $\mathrm{A}$ and Theorem B.

\section{References}

[1] C P Boyer, B M Mann, Monopoles, nonlinear $\sigma$ models, and two-fold loop spaces, Comm. Math. Phys. 115 (1988) 571-594 MR933456

[2] F R Cohen, R L Cohen, B M Mann, R J Milgram, The topology of rational functions and divisors of surfaces, Acta Math. 166 (1991) 163-221 MR1097023 
[3] F R Cohen, R L Cohen, B M Mann, R J Milgram, The homotopy type of rational functions, Math. Z. 213 (1993) 37-47 MR1217669

[4] Y Kamiyama, Polynomial model for homotopy fibers associated with the James construction, Math. Z. 237 (2001) 149-180 MR1836776

[5] J Mostovoy, Spaces of rational loops on a real projective space, Trans. Amer. Math. Soc. 353 (2001) 1959-1970 MR1813601

[6] G Segal, The topology of spaces of rational functions, Acta Math. 143 (1979) 39-72 MR533892

[7] V A Vassiliev, Complements of discriminants of smooth maps: topology and applications, Translations of Mathematical Monographs 98, American Mathematical Society, Providence, RI (1992) MR1168473 Translated from the Russian by B Goldfarb

Department of Mathematics, University of the Ryukyus

Nishihara-Cho, Okinawa 903-0213, Japan

kamiyama@sci.u-ryukyu.ac.jp

Received: 13 August 2004 Revised: 9 May 2005 